\title{
Improving Packet Delivery Efficiency Using Dual 3GPP LTE Communication Modules for Railroad Wireless Communication
}

\author{
Ronny Yongho Kim and Baik Kim* \\ Department of Railroad Electrical and Electronics Engineering, \\ Korea National University of Transportation, \\ Uiwang, Gyeonggi, 437-763 Korea \\ whitek@ut.ac.kr
}

\begin{abstract}
As a next generation of Global System for Mobile Communications - Railway (GSM-R), 3rd Generation Partnership Program (3GPP) Long Term Evolution (LTE)/ LTE-Advanced (LTE-A) is considered for railroad wireless communication, i.e., LTE-Railway (LTE-R). Railroad wireless communication has its own requirements and functions since railroad wireless communication is very closely related to safety. In order to meet the requirements of LTE-R and provide high QoS level, it is very crucial to have very small packet delivery delay. Since it is anticipated that it will be difficult to deploy LTE-R network with redundant network architecture like GSM-R due to spectrum shortage, high QoS and reliability of LTE communication is very important for LTE-R. Railroad wireless communication network configuration has very unique characteristics compared to other wireless communication networks. Since train moves on pre-installed fixed track, train movement can be easily measured. In this paper, by exploiting the unique characteristic of railroad wireless communication, an improved packet delivery scheme using dual communication modules for railroad wireless communication is proposed. By using the proposed scheme, almost zero service interruption time during handover can be achieved. Since the protocol architecture of the proposed scheme utilizes the current LTE railroad wireless communication hardware, the proposed scheme can be utilized with minimum modification.
\end{abstract}

Keywords: We would like to encourage you to list your keywords in this section

\section{Introduction}

Railroad transportation is getting more attention than ever since it is able to provide environment friendly operation with substantially less carbon dioxide emission compared to other transportations. In order to provide better railroad services, high Quality of Service (QoS) level railroad communication is essential since train control system, safety provision system, communication system and passenger service system are realized using railroad communication system. Variety of wireless technologies, e.g., Terrestrial Trunked Radio (TETRA) [1], GSM-R [2], IEEE 802.11 [3], etc. are used for railroad communications. In order to provide high quality railroad communications, service interruption time due to handover needs to be substantially reduced.

One of the 4G wireless technologies, 3GPP LTE/LTE-A has been considered to be used for railroad communications as LTE-R [4] which is a next generation of GSM-R.

\footnotetext{
${ }^{*}$ Baik Kim is the corresponding author.
} 
International Union of Railways (UIC) has commenced to generate LTE-R requirements [5]. LTE-A provides high QoS level services meeting and most cases exceeding the 4G requirements of ITU-R. In order to provide advanced services, LTE-A provides various functions and features including mobile relay, small cell technology and carrier aggregation. Carrier aggregation is one of representative technology of LTE-A providing high throughput.

Though 4G wireless technology including 3GPP LTE [6] is designed to provide high QoS level, its performance under all possible railroad operation environments needs to be fully investigated since thousands of railroad passengers' lives could depend on the reliability of railroad communication.

This paper proposes a packet delivery delay reduction scheme in railroad wireless communication system using LTE. The proposed scheme utilizes the unique characteristic of railroad wireless communication network configuration. Typically used railroad LTE communication module can be easily extended to support the proposed scheme. By using the proposed scheme, almost zero service interruption time during handover can be achieved. A preliminary result of this paper was presented in [7].

The remaining part of the paper is organized as follows. In Section 2, overview on railroad wireless communication using 3GPP LTE from network configuration and packet transmission delay point of view is presented. Description on the proposed packet delivery scheme is provided in detail in Section 3. Finally, we conclude the paper in Section 4.

\section{Railroad Wireless Communication Using 3GPP LTE}

Railroad wireless communication system has unique network configuration in comparison with other human communication networks since train moves along with the fixed railroad. In this section, description on railroad wireless communication network using LTE and LTE packet transmission delay during handover is provided.

\subsection{Railroad wireless communication network configuration using LTE}

Railroad communication network has its unique network configuration since train moves along with fixed railroad. A train which consists of multiple train cars moves along fixed train track. Since railroad communication network is a linear network and train's movement is prescheduled, communication of train passengers or crews can be easily managed. If such unique characteristics of railroad wireless communication network configuration are properly utilized, very efficient communication schemes can be designed.

Railroad user communication is to provide railroad passengers with communication services. Railroad users, LTE User Equipments (UEs) are able to communicate either directly with base station or indirectly via train communication devices such as WiFi Access Point (AP) and on-board terminal. The common way for train passengers to access data is using WiFi installed in railroad cars. In order to provide a good quality of broadband wireless services to train passengers connected using WiFi, typically on-board cellular terminal's structure is configured with multiple LTE UEs which are utilized to provide load balancing among multiple passengers' WiFi connections.

LTE/LTE-Advanced can be simply extended to be used for railroad communication. Such LTE system used for railroad is called LTE-R which is a next generation of GSM-R. By extending and adding railroad specific functions and services to basic railroad wireless communication network configuration using LTE, railroad systems such as train control system, safety provision system, communication system, passenger service system, and etc. can be realized over LTE-R network. The main benefit of using LTE-R is that various railroad related aforementioned systems can be implemented using a single LTE-R network 
since LTE/LTE-A is a broadband wireless communication system providing high QoS level Internet Protocol (IP) data communication.

LTE-R core network is expected to be almost same as LTE/LTE-A network except some IP Multimedia Subsystem (IMS) functional extension for railroad centric services such as group Voice over LTE (VoLTE), high priority call processing and etc.

\subsection{Packet transmission delay problem during handover}

In cellular network, as a mobile terminal moves among cells, a procedure called handover should be performed in order to provide continuous services. However there is some service interruption time due to requiring actions for a serving base station (BS) change such as radio frequency change including synchronization and handover control message exchange. Figure 1 shows a basic X2 interface based handover procedure. In order to trigger a handover, the serving eNB requests the UE to report radio link quality measurement. When a radio link quality of the serving eNB is below a handover threshold and a radio link quality of the target eNB is above a handover threshold, handover decision is made by the serving eNB.

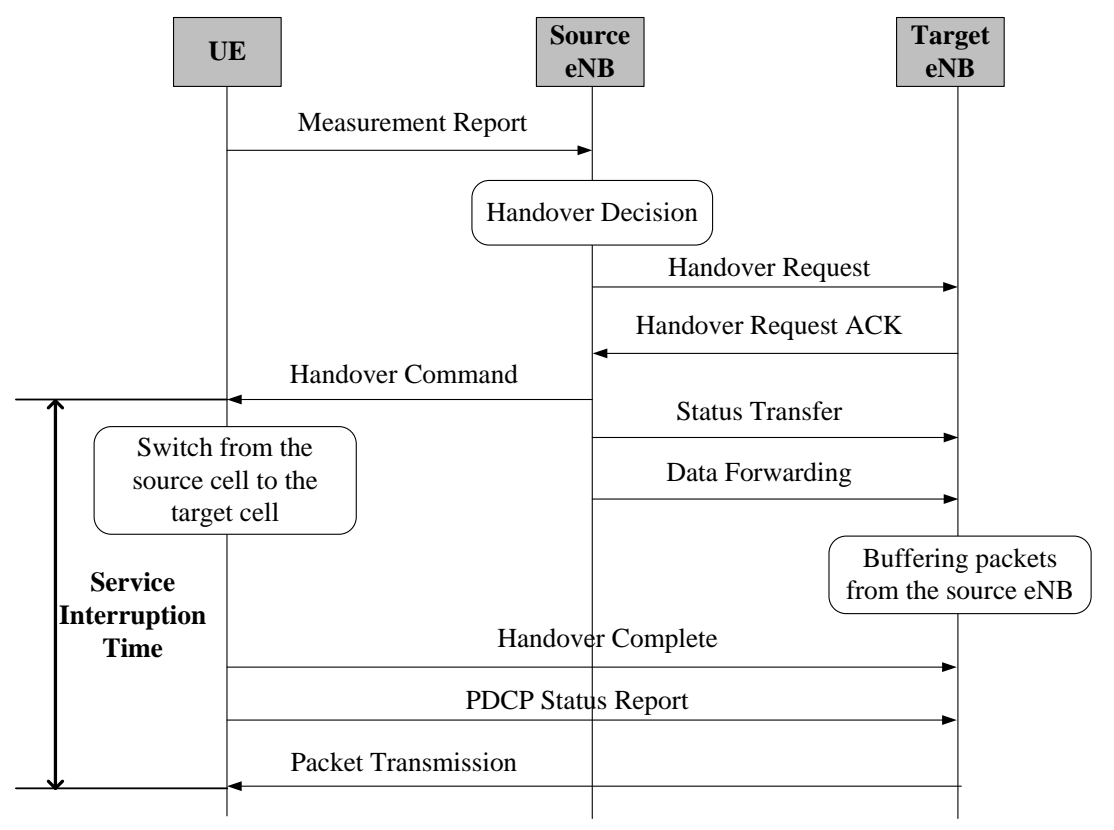

\section{Figure 1. LTE Basic X2 Interface Based Handover Procedure}

In 3GPP LTE, if eNBs are connected to a same Mobility Management Entity (MME) [8], eNBs can be interconnected to each other through direct interface called X2 interface. By utilizing direct interface between eNBs, handover interruption time of LTE is much less than previous 3GPP wireless systems.

In order to provide efficiency and robustness in data packet transmission, Packet Data Convergence Protocol (PDCP) layer is used in 3GPP [9]. PDCP provides header compression using Robust Reader Compression (ROHC) [10], security functions, maintenance of PDCP sequence numbers, timer-based Service Data Unit (SDU) discard and so on. During handover, the PDCP layer as well as Radio Link Control (RLC) layer is re-established upon request from the Radio Resource Control (RRC) layer. In 3GPP LTE, Acknowledgement Mode (AM) Data Resource Block (DRB) is used to support lossless transmission. Lossless transmission is normally guaranteed by the AM RLC entity in normal situations. In case of handover, PDCP 
status report is used for lossless transmission. Therefore, by transmitting PDCP status report after handover, lossless transmission can be guaranteed without duplicate packet transmission.

\section{Improved Packet Transmission Scheme using Dual Communication Modules}

\subsection{Proposed protocol architecture}

Typically multiple LTE communication modules (UEs) are configured in an on-board terminal. For simplicity, an on-board terminal with two LTE communication modules (UEs) is used in our example of Figure 2. If the unique characteristic of railroad communication is considered, we can fully exploit multiple LTE connections. As we can see from Figure 3, even though front car of the train entered into the target eNB coverage area, rear car might still have good wireless link connection with the source eNB. If antennas are installed on the front most car and the rear most car and connected to two different LTE communication modules (UEs), very efficient packet delivery delay reduction scheme can be designed.

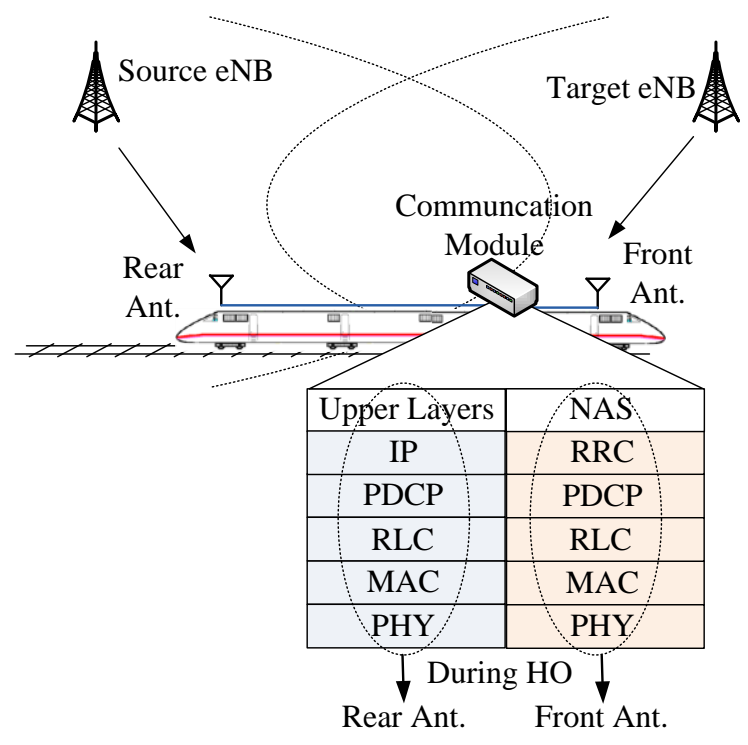

Figure 2. Proposed Protocol Structure for a Proposed Minimum Packet Delay Transmission Scheme

When a handover procedure is triggered, the LTE communication module using the front most antenna performs handover procedure with control plane protocol layers: RRC, PDCP, RLC, MAC and PHY. While performing a handover procedure, the LTE communication module using the rear most antenna maintains the LTE wireless link with the source eNB and continuously receives the packets. In normal handover procedure, when a handover procedure is commenced, no packet is transmitted to the UE until the handover completion. By using the proposed packet transmission scheme, almost zero service interruption time with minimized packet delivery delay can be achieved.

\subsection{Proposed procedure using the proposed scheme}

Figure 3 shows the proposed packet transmission procedure using the proposed scheme. Protocol architecture described in the previous section is used to describe the proposed packet 
transmission procedure. Basic handover procedure is same as the one explained in Section 2 using Figure 1. The main benefit of our proposed scheme comes from the unique network configuration of railroad wireless communication. Since one of the LTE communication modules' antennas is installed on the rear most car, while the other LTE communication module is performing handover procedure with the target eNB, the LTE communication module can still receive packets from the source eNB. After handover, PDCP layer of UE transmits PDCP status report to the target eNB. The target eNB utilizes the received PDCP status report to exclude the positively acknowledged packets by the UE.

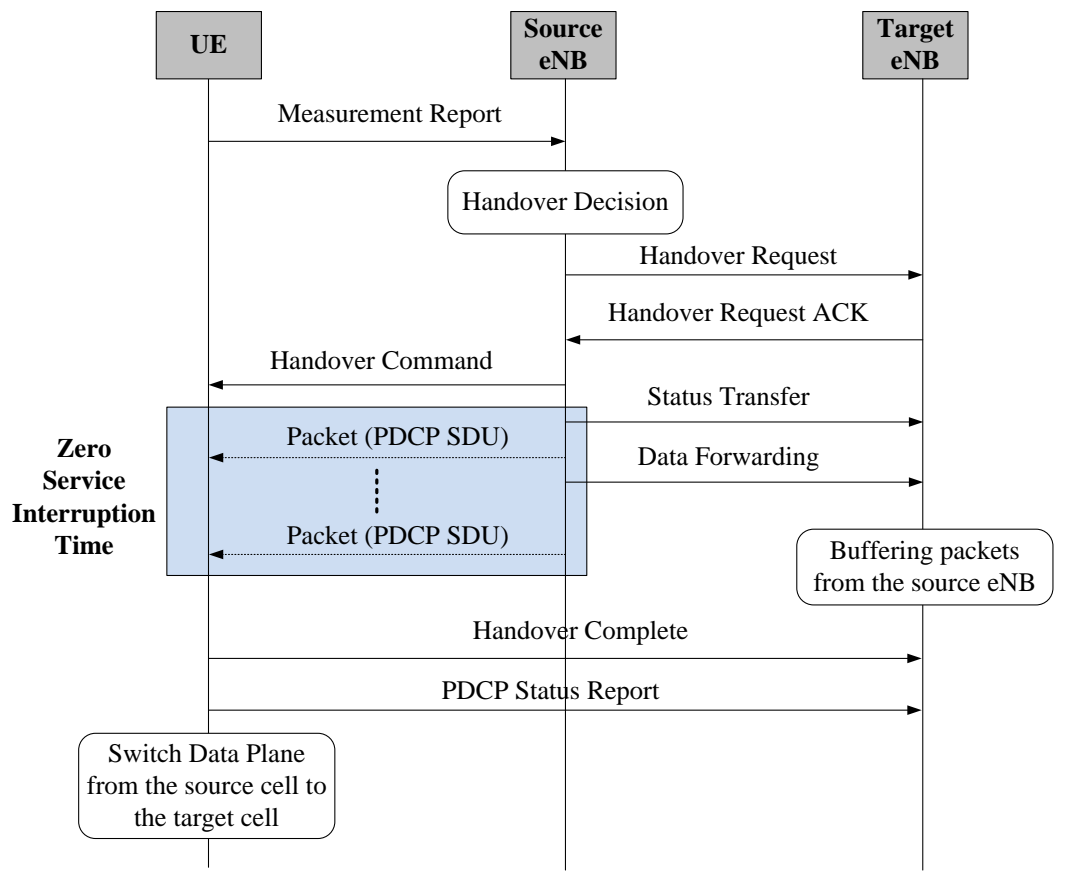

\section{Figure 3. Proposed Packet Transmission Procedure using the Proposed Scheme}

\section{Conclusion}

In this paper, an improved packet delivery scheme for railroad wireless communication system using dual 3GPP LTE communication modules is proposed. The proposed scheme exploits the unique characteristic of railroad wireless communication. In order to minimize the impact on the LTE standard, existing handover procedure is intact and minor modification is made to accommodate additional packet transmission from the source eNB. Protocol architecture is designed based on the currently used railroad LTE hardware. By using the proposed scheme, almost zero service interruption time during handover can be achieved. Since the protocol architecture of the proposed scheme utilizes the current LTE railroad wireless communication hardware, the proposed scheme can be utilized in practice with minimum modification.

\section{Acknowledgements}

This research was supported by Basic Science Research Program through the National Research Foundation of Korea(NRF) funded by the Ministry of Education, Science and Technology(NRF-2012R1A1A1014610). 


\section{References}

[1] Terrestrial Trunked Radio, "Voice plus Data (V+D), Part 2: Air Interface (AI)", ETSI EN 300 39202, vol. 3, no. 2, (2007).

[2] UIC, "GSM-R Procurement Guide," (2007) February.

[3] "IEEE Standard for Information technology--Telecommunications and information exchange between systems Local and metropolitan area networks--Specific requirements Part 11: Wireless LAN Medium Access Control (MAC) and Physical Layer (PHY) Specifications”, IEEE Std, vol. 802, no.11, (2012).

[4] 1st Year Research Report on Railroad Wireless Communication System, Korea Railroad Research Institute, (2011).

[5] www.uic.org.

[6] 3GPP Technical Specification 36.300, "E-UTRA and E-UTRAN Overall Description Stage 2 (Release 10)", www.3gpp.org.

[7] R. Y. Kim and B. Kim, "Packet delivery delay reduction scheme in railroad wireless communication system using 3GPP LTE," Proceedings of the Workshop on Networking and Communication, Jeju, Korea, (2013) December 11-13.

[8] 3GPP Technical Specification 23.002, "Network architecture (Release 12)", www.3gpp.org.

[9] 3GPP Technical Specification 36.323, "E-UTRA Packet Data Convergence Protocol (PDCP) (Release 11)", www.3gpp.org.

[10] IETF RFC 4995 - "The RObust Header Compression (ROHC) Framework”, (2007) July.

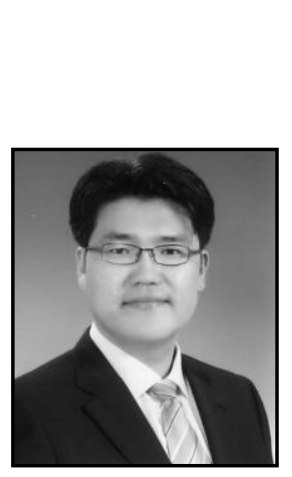

\section{Authors}

Ronny Yongho Kim received his Ph.D. degree from Yonsei University, Seoul, Korea, 2010. He became an IEEE Senior Member in 2010. From 1998 to 2010, he was with LG Electronics. Since 2012, he has been with the Department of Railroad Electrical and Electronics Engineering, Korea National University of Transportation, Gyeonggi, Korea, where he is an Assistant Professor. His current research interests are railroad communications, wireless networks, IT convergence and machineto-machine communications.

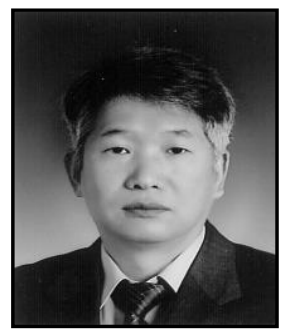

Baik Kim received his Ph.D. degree from Yonsei University, Seoul, Korea, 1995. From 1980 to 1996, he was with the Korea Electric Power Corporation, Korea. Since 1996, he has been with the Department of Railroad Electrical and Electronics Engineering, Korea National University of Transportation, Gyeonggi, Korea, where he is a Professor. His current research interests are railroad engineering, power systems and computer science. 